\title{
All-flavour high-energy neutrino astronomy with KM3NeT/ARCA
}

\author{
Paolo Piattelli* \\ for the KM3NeT Collaboration \\ INFN - Laboratori Nazionali del Sud, Catania (Italy) \\ E-mail: piattellielns.infn.it
}

\begin{abstract}
The recent discovery by the IceCube collaboration of a high-energy neutrino flux of extraterrestrial origin has opened a new observational window on the Universe. However, unambiguous identification of the emitting neutrino sources will require next generation neutrino telescopes with full sky coverage. The KM3NeT Collaboration aims at building a research infrastructure in the depths of the Mediterranean Sea hosting a multi-cubic-kilometre neutrino telescope. The technology for the detector construction and operation is defined and under final test with prototypes at depths of $2500 \mathrm{~m}$ and $3500 \mathrm{~m}$. The detector array of optical modules will be subdivided into sizeable building blocks of string-type detection units. This intrinsically modular nature of the detector allows for a staged implementation with increasing size from about $0.1 \mathrm{~km}^{3}$ for highenergy astronomy (phase-1) to be completed by 2016 to a volume of several $\mathrm{km}^{3}$ in the final phase. The following phase, KM3NeT 2.0, will comprise two detectors with different granularity of optical modules: KM3NeT/ARCA at the KM3NeT-It site offshore Capo Passero (Italy) with two building blocks dedicated to high-energy neutrino astronomy and KM3NeT/ORCA, a single building block located at the KM3NeT-Fr site offshore Toulon (France) dedicated to the study of neutrino mass hierarchy. The latitude of KM3NeT/ARCA will allow for a wide coverage of the observable sky including the region of the galactic centre. Owing to the favourable characteristics of sea water the direction of neutrinos will be measured with excellent angular resolution also for cascade events. The expected KM3NeT/ARCA sensitivity will allow for the detection of the reported IceCube flux within less than one year of observation, providing new insight on its origin, energy spectrum and flavour composition. Within five years of observation KM3NeT/ARCA could give indications at $3 \sigma$ level on various galactic point-like sources.
\end{abstract}

The 34th International Cosmic Ray Conference,

30 July- 6 August, 2015

The Hague, The Netherlands

\footnotetext{
* Speaker.
} 


\section{Introduction}

KM3NeT will be a new Research Infrastructure composed of a network of deep-sea installations in the Mediterranean Sea and hosting the largest telescope for high-energy neutrinos.

The ultimate goal of KM3NeT is to construct a neutrino telescope with at least $3 \mathrm{~km}^{3}$ of instrumented volume; an implementation as a distributed multi-site facility is envisaged. The recent IceCube discovery of a flux of cosmic neutrinos $[1,2]$ and the reported non-zero value for the neutrino mixing angle $\theta_{13}$ have strongly motivated the realization of an intermediate phase: KM3NeT 2.0. It will consist of two detectors with different granularity: KM3NeT/ARCA, with two building blocks installed at the KM3NeT-It site and dedicated to high-energy neutrino astronomy, and KM3NeT/ORCA [3], with a single building block installed at the KM3NeT-Fr site and dedicated to the study of the neutrino mass hierarchy. The two detectors will share the same technology with a centralized management and a unique data centre.

The main objective of KM3NeT/ARCA is the discovery of high energy neutrino sources in the cosmos. Neutrinos are a powerful probe for high energy astrophysics since, thanks to their faint interaction probability, they can propagate from the astrophysical sources to the Earth preserving their initial direction and energy. They can therefore carry unperturbed information on the high energy particle acceleration mechanisms in the cosmos. On the other hand they are extremely difficult to detect and cubic-kilometer detectors with a very low background level are required.

Starting in 2004 as a coherent effort of the Mediterranean neutrino telescope collaborations (ANTARES, NEMO and NESTOR), the KM3NeT collaboration has developed a cost-effective technology for the construction of an underwater neutrino telescope and reached now the stage to start its construction. Due to its location in the Mediterranean Sea, KM3NeT will be in an optimal position to investigate neutrino fluxes from the Southern sky, in particular from the galactic centre and from a large fraction of the galactic plane, thus complementing the field of view of the IceCube detector. Investigating the properties and the sources of the IceCube flux is a main objective of KM3NeT/ARCA. The next challenge is the identification of point-like neutrino sources. KM3NeT/ARCA will be able to give indication at $3 \sigma$ level on some of these objects in about five years of operation.

\section{The KM3NeT/ARCA neutrino telescope}

The detection principle of KM3NeT is based on the measurement of the Cherenkov light induced in water by the secondary charged particles produced in neutrino interactions inside or in the vicinity of the detector. The arrival times of the light collected by optical sensors and their positions are used to reconstruct the secondary particles. From these the neutrino arrival directions can be inferred and their energies estimated.

The basic unit of the KM3NeT detector is the Digital Optical Module (DOM) [4]. The DOM is a pressure-resistant glass sphere of 17-inch diameter containing 31 3-inch photomultiplier tubes (PMTs) with their associated electronics as well as calibration instrumentation. Nineteen of these PMTs are located in the lower hemisphere, and are thus looking downwards, while 12 in the upper hemisphere are looking upwards (Fig. 1). This novel design offers significant improvements with respect to optical modules with a single large-area PMT: the total photocatode area is about three 


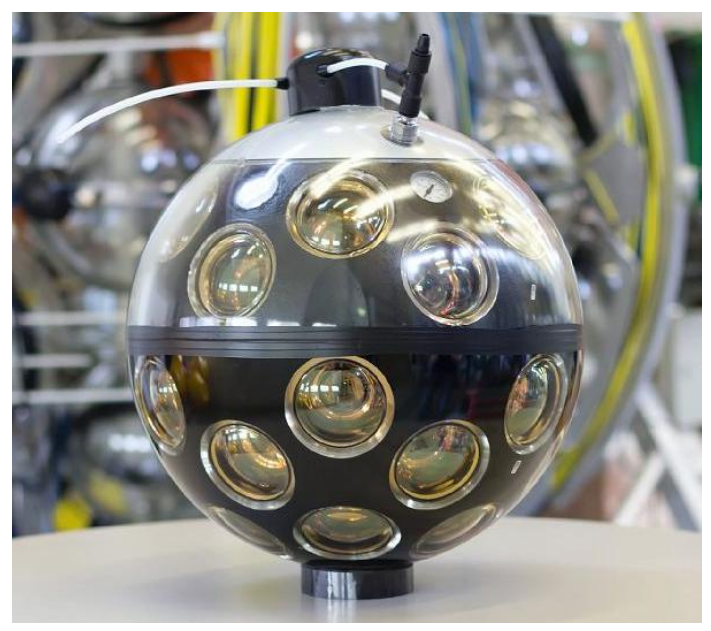

Figure 1: The KM3NeT Digital Optical Module.

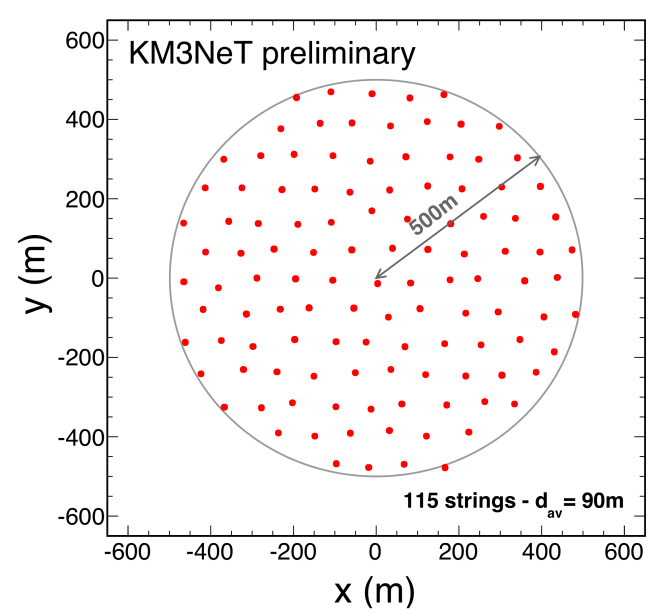

Figure 2: Footprint of one block of the KM3NeT/ARCA telescope. The detector will be composed by two of such blocks

times larger, the field of view covers almost the full solid angle, pixelisation allows for high-purity photon counting and directional sensitivity. All the necessary electronics for digitization and data transmission is contained within each DOM.

A sequence of 18 DOMs, vertically spaced by $36 \mathrm{~m}$, forms a Detection Unit (DU) [5]. Two thin parallel ropes hold the DOMs in place. The DU is anchored on the seabed and kept taut by a submerged buoy at its top. A vertical "backbone" cable, running along the full length of the DU, provides connectivity for power and data transmission. This cable is an oil-filled plastic tube, in equi-pressure with sea water, containing electrical wires and optical fibres, with a breakout box at each DOM. For the deployment the DU is coiled on a spherical aluminum frame with $2 \mathrm{~m}$ diameter, called Launcher of Optical Modules (LOM). In this compact configuration the DU is deployed on the seabed and connected to the seafloor network. The LOM is then released, unfurling the DU while rotating upwards. It floats to the surface where it is recovered for further use [5].

A set of $115 \mathrm{DU}$, spaced with an average distance of $90 \mathrm{~m}$ and arranged with a roughly circular footprint, forms a "building block" (Fig. 2). KM3NeT/ARCA will comprise two of such building blocks for a total in instrumented volume of about $1 \mathrm{~km}^{3}$. The DUs are connected to junction boxes and finally to shore by means of a main electro-optical cable. The architecture is intrinsically modular allowing for a staged implementation of the telescope.

\section{Present status and plans}

The prototyping phase of KM3NeT, initiated within a Preparatory Phase co-funded by the EU, has included two main steps [6]. A first DOM prototype has been installed in the deep sea in April 2013 in the ANTARES Instrumentation Line, which is connected to shore via a $45 \mathrm{~km}$ fiber-optic link. The analysis of the data, reported in [7], verified the full functionality of the DOM concept and in particular also demonstrated its capability to identify muon signals by selecting 
high-fold coincidences between the PMTs of the same DOM. In May 2014, a prototype detection unit housing three optical modules was deployed offshore Portopalo di Capo Passero (Italy) at 3500 $m$ depth with the main aim of testing the string concept and deployment technique as well as interDOM synchronisation, readout, data acquisition and the connection to the deep-sea cable network. This prototype has been operated continuously since its deployment proving the functionality and reliability of the concept. The data analysis confirms the specifications of the system. By selecting coincidences between couples of DOM it was possible to identify atmospheric muon signals. InterDOM calibration and its stability have been checked both with fast light pulsers installed in each DOM and with the muon signals [6].

Following the successful prototyping phase, the KM3NeT collaboration is pursuing the construction of a phase-1 detector at the KM3NeT-Fr and KM3NeT-It sites. Production, deployment and connection of 31 DUs will take place in the period 2015 to 2016. The first DU is ready to be deployed at KM3NeT-Fr site and will serve as a concluding engineering test. Twenty-four DUs will be installed in the KM3NeT/ARCA configuration at the KM3NeT-It site. The remaining six DUs will be realized with a more compact configuration as a test for the KM3NeT/ORCA detector and will be installed at the KM3NeT-Fr site. Already in this first configuration the first stage of KM3NeT/ARCA will be the most sensitive neutrino telescope in the Northern hemisphere, with roughly 3 times the ANTARES sensitivity.

The next objective is the construction of KM3NeT 2.0 with two installations: KM3NeT/ORCA and KM3NeT/ARCA at the French and Italian sites, respectively. With two full building blocks, KM3NeT/ARCA will provide a sensitivity similar to that of IceCube. This installation, which could be completed around 2017/18 if funding is secured, will have as a entral physics focus the investigation of the characteristics and sources of the cosmic neutrino flux detected by IceCube. Full KM3NeT construction, with six ARCA building blocks, is foreseen to proceed subsequently.

\section{Performance and physics objectives of KM3NeT/ARCA}

The main physics cases for KM3NeT/ARCA are the search for diffuse neutrino fluxes, in particular the investigation from a complementary field of view and with better angular resolution of the IceCube findings [1,2], and the search for point-like neutrino sources, with a particular focus on the galactic ones.

Evaluation of the telescope performance has been carried out using complete Monte Carlo simulation, including the neutrino interaction in the medium, the propagation of the resulting secondary particles, the Cherenkov light generation and propagation in water and the detector response. Depth and the optical water properties measured at the KM3NeT-It site have been used [8]. Background due to the presence of ${ }^{40} \mathrm{~K}$ in salt water was simulated adding an uncorrelated hit rate of $5 \mathrm{kHz}$ per PMT plus higher-fold coincidence rates as determined by GEANT simulations and in agreement with the results from the prototype optical modules.

Backgrounds due to downgoing atmospheric muons and to neutrinos originating in cosmic-ray interactions in the atmosphere have also been taken into account in the simulations.

KM3NeT/ARCA is sensitive to all neutrino flavors, since events of different topology can be detected and identified. Track like events, generated mainly by $v_{\mu}$ Charged Current (CC) interactions, constitute the "classic" detection channel. These events can be generated far from the 

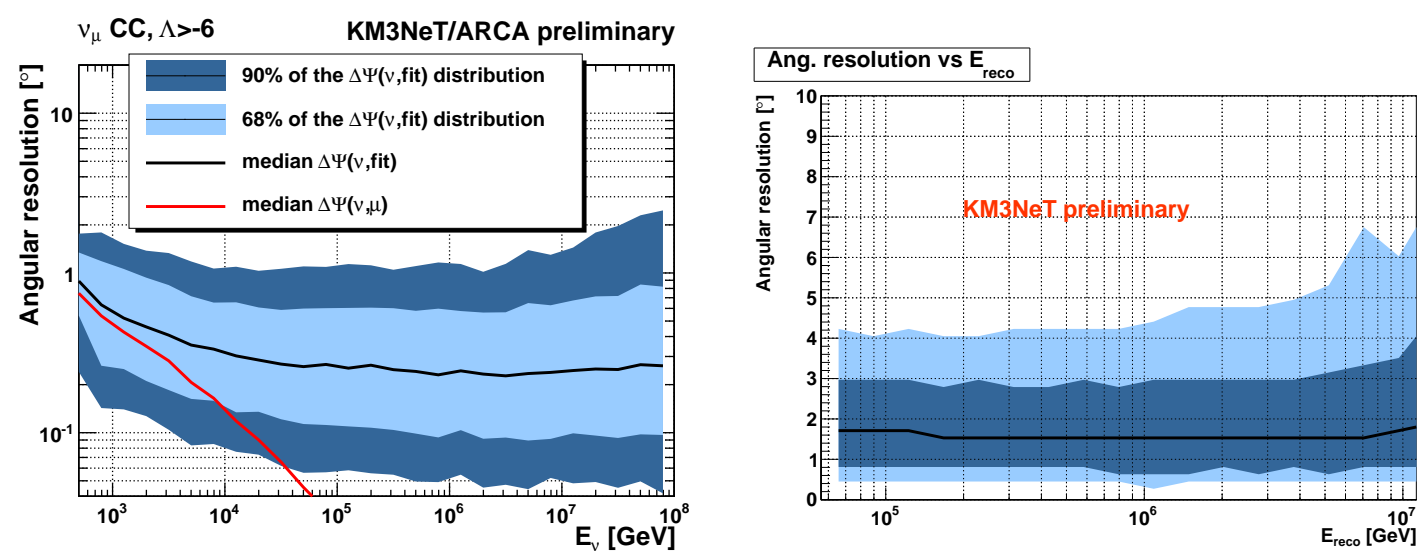

Figure 3: KM3NeT/ARCA neutrino angular resolution as a function of neutrino energy for track-like (left panel) and shower-like (right panel) events. Events are after an event selection cut according to KM3NeT/ARCA sensitivity analysis for point-like (left) and diffuse (right) fluxes. Light and dark blue areas correspond to the $68 \%$ and $90 \%$ quantile respectively.

detector, as high-energy muons can travel several kilometers in water. The identification of these events is however limited to up-going tracks due to the presence of the atmospheric muon background. Shower-like events, such as those generated in $v_{e}$ Charged Current (CC) and in Neutral Current (NC) interactions can also be detected, provided that the interaction occurs inside or close to the detector volume.

A reconstruction technique for muon tracks that fully exploits the DOM capabilities has been developed [9]. It reconstructs the muon trajectory by maximizing the likelihood that the expected space-time hit pattern comes from the corresponding Cherenkov light emission. A very good angular resolution of about $0.2^{\circ}$ (left panel in Fig. 3) and an energy resolution of $28 \%$ in $\log \left(E_{\mu}\right)$ are achieved for neutrino energies above $10 \mathrm{TeV}$.

For high-energy events $(E>50 \mathrm{TeV})$ the shower reconstruction reaches an accuracy on the vertex reconstruction of about $1 \mathrm{~m}$, an angular resolution of $2^{\circ}$ (right panel in Fig. 3) and an energy resolution of roughly $10 \%$.

\subsection{KM3NeT/ARCA sensitivity to neutrino diffuse fluxes}

Both track and shower analyses have been used to investigate the sensitivity of KM3NeT/ARCA to the neutrino flux reported by IceCube. In this analysis an isotropic one-flavour flux $\Phi=$ $1.2 \times 10^{-8}(E / 1 \mathrm{GeV})^{-2} e^{-\left(E_{v} / 3 \mathrm{PeV}\right)} \mathrm{GeV}^{-1} \mathrm{~cm}^{-2} \mathrm{~s}^{-1} \mathrm{sr}^{-1}$, as reported in [1], is assumed. Results are reported in [11] and summarized here in Fig. 4.

For the track channel, up-going muon tracks are selected applying cuts on the reconstructed muon zenith angle $\left(\theta_{z}>80^{\circ}\right)$ and on a reconstruction quality parameter to suppresss most of the atmospheric muon background. To reduce the atmospheric neutrino background, a cut on the number of hits, which is correlated with the neutrino energy, is also applied. A significance of $5 \sigma$ is reached after less than 2 years. The major uncertainty for this channel (shown as a shaded area in the figure) is represented by the uncertainty on the conventional atmospheric neutrino flux.

The shower channel gives a much better sensitivity, reaching a $5 \sigma$ significance after less than one year. In this analysis, background due to atmospheric muons can be rejected applying geomet- 


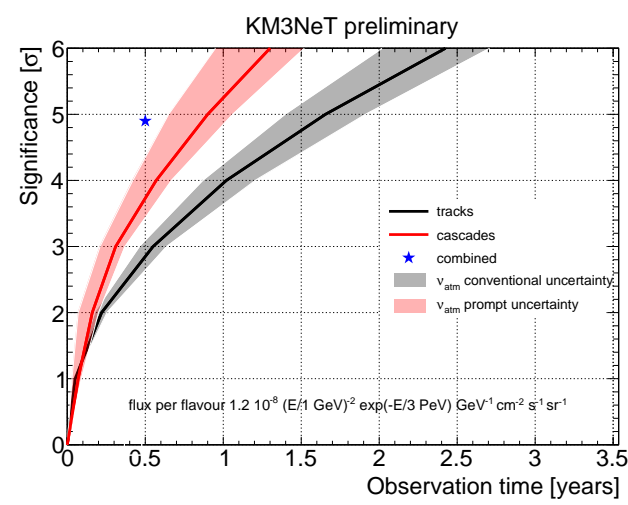

Figure 4: Significance as a function of the observation time for the detection of a diffuse flux of neutrinos corresponding to the signal reported by IceCube, in the up-going muon channel (black), the cascade channel (red) and the combined analysis (blue star). Shaded areas indicate the maximum values of uncertainties.

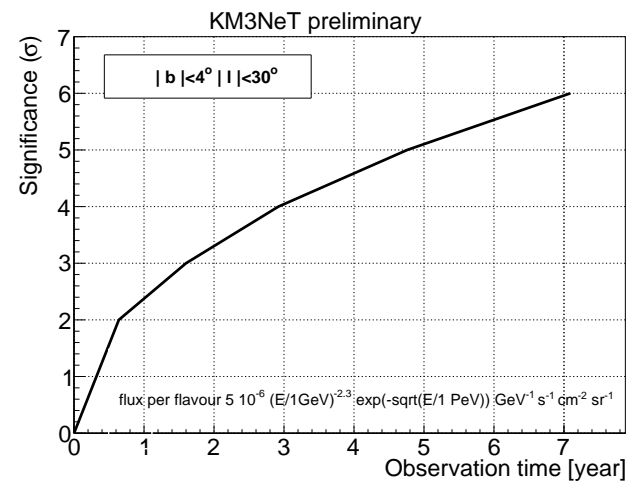

Figure 5: Significance as a function of the observation time of the diffuse neutrino flux predicted in [14] in the up-going muon channel.

rical cuts on the reconstructed interaction vertex (containment) and by an energy cut applied using the time over threshold information of the PMTs, which is correlated to the charge collected. The final step to select signal events is performed by a machine learning algorithm. The major contribution to systematic uncertainty for this channel is due to the uncertainty on the prompt neutrino flux.

A combined analysis, incorporating the results from both search strategies has also been developed, giving as a final result a significance of $4.8 \sigma$ in 0.5 years.

A guaranteed source of high energy neutrinos is the galactic plane region. The fact that several of the detected IceCube neutrinos come from close to the galactic plane region and the galactic centre has stimulated the phenomenological investigation of the neutrino flux from the interaction of cosmic rays with the interstellar medium. In this scenario several works have recently appeared $[12,13,14]$ where the high-energy neutrino and the high-energy gamma-ray fluxes were estimated and compared with the existing data. In particular in $[12,14]$ the gamma and neutrino emission was estimated with the cosmic ray diffusion model where a radial dependence of the diffusion coefficient was assumed. In this framework the neutrino flux from a limited region of the galactic plane near the galactic centre $\left(|b|<4^{\circ},|l|<30^{\circ}\right.$; with $b$ and $l$ being the galactic latitude and the galactic longitude respectively) has been estimated and reported in [14].

With a similar analysis as described in [11] for the track channel the preliminary performances of KM3NeT/ARCA to this flux has been estimated and shown in Fig. 5. A significance of $5 \sigma$ is reached in about 5 years.

\subsection{Sensitivity to point-like sources}

The location in the Mediterranean Sea makes the KM3NeT/ARCA detector a powerful tool to explore and identify galactic neutrino sources. The detector lay-out used for Monte Carlo simula- 


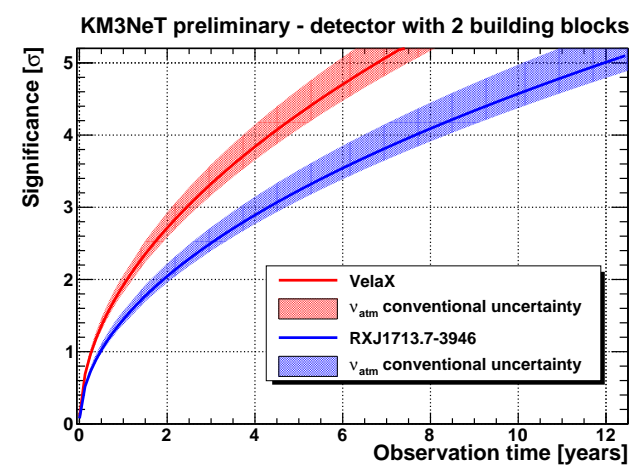

Figure 6: Significance of the RXJ1713.7-3946 and Vela-X observation as a function of the years of data taking for the KM3NeT/ARCA detector. The shaded areas show the effect of the uncertainties on the conventional component of the atmospheric neutrino flux.

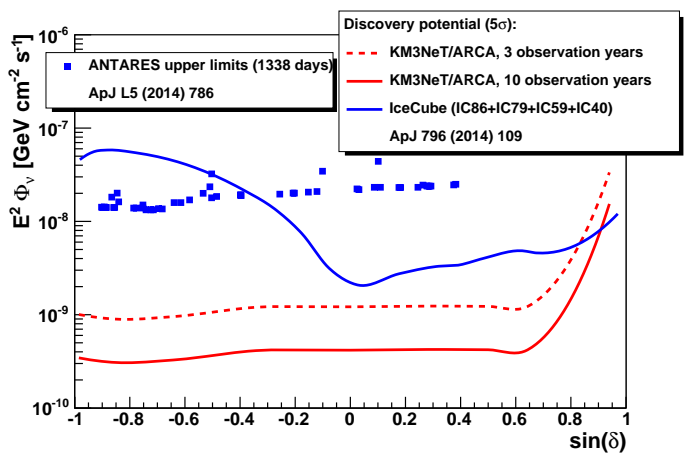

Figure 7: KM3NeT/ARCA $5 \sigma$ discovery potential per flavor for point sources emitting a neutrino flux with a $E^{-2}$ spectrum, for 3 and 10 years of data taking. The flux values are shown as function of the source declination. For comparison the corresponding IceCube discovery potential to sources with an $E^{-2}$ spectrum [20] and the upper limits quoted by ANTARES [21] are also shown.

tions has been optimized for the detection of galactic sources using as a test case the very intense SuperNova Remnant gamma source RXJ1713.7-3946 [15] and the Pulsar Wind Nebula Vela-X [16]. The neutrino energy spectrum was estimated from the gamma-ray spectrum assuming a $100 \%$ hadronic mechanism and a source transparent to gamma-ray emission according to [17] for RXJ1713.7-3946 and [18] for Vela-X.

Under these assumptions, the significance of the source observation, with a 50\% probability, as a function of the observation time for KM3NeT/ARCA has been calculated [19] and is shown in Fig. 6. An observation with $3 \sigma$ significance is expected after about 2.5 and 4 years for Vela-X and RXJ1713.7-3946, respectively. The extension of the KM3NeT telescope to its final configuration of six blocks will allow to reach a $5 \sigma$ significance after about 2.5 years for Vela-X and 4 years for RXJ1713.7-3946.

The $5 \sigma$ discovery flux has also been calculated for a generic point-source with an $E^{-2}$ spectrum as a function of the declination (Fig. 7) for 3 years of observation time. This is a similar exposure as used for the IceCube discovery flux reported in [20] that is also shown in Fig. 7 for comparison. The different trends of the KM3NeT and IceCube curves are mainly due to the geographical location of the detectors.

\section{Conclusions}

As recently impressively demonstrated by the first detection of high-energy cosmic neutrinos with the IceCube detector, neutrino telescopes can be sensitive to neutrinos of all flavours and from all directions, albeit not equally for all reaction channels and energies.

Following a successful design and prototyping phase, the KM3NeT collaboration is presently engaged in the construction of a phase- 1 detector at two sites: one in Italy and one in France. The 
following phase, KM3NeT 2.0, will comprise two detectors with different granularity of optical modules: KM3NeT/ARCA at the KM3NeT-It site, with two building blocks dedicated to highenergy neutrino astronomy, and KM3NeT/ORCA, a single building block located at the KM3NeTFr site offshore Toulon (France) dedicated to the study of neutrino mass hierarchy.

Due to its location in the Mediterranean Sea, KM3NeT/ARCA is an optimal position to investigate neutrino fluxes from the Southern sky, in particular from the galactic centre and the largest fraction of the galactic plane. The expected KM3NeT/ARCA sensitivity will allow for the detection of the reported IceCube flux within less than one year of observation, providing new insight on its origin, energy spectrum and flavour composition. Within five years of observation KM3NeT/ARCA will also be able to give indications at $3 \sigma$ level on some of the most intense galactic sources.

\section{References}

[1] M. G. Aartsen et al. (IceCube Collaboration), Science 342 (2013) 1242856.

[2] M. G. Aartsen et al. (IceCube Collaboration), Phys. Rev. Lett. 113 (2014) 101101.

[3] J. Brunner for the KM3NeT collaboration, proceedings of this conference ID1140.

[4] R. Bruijin et al. for the KM3NeT Collaboration, proceedings of this conference ID1157.

[5] P. Kooijman et al., for the KM3NeT Collaboration, proceedings of this conference ID1173.

[6] S. Biagi et al., for the KM3NeT Collaboration, proceedings of this conference ID1164.

[7] S. Adrian-Martinez et al. (KM3NeT Collaboration), Eur. Phys. J. C 74 (2014) 3056.

[8] G. Riccobene et al., Astropart. Phys. 27 (2006) 1.

[9] A. Trovato et al., for the KM3NeT Collaboration, proceedings of this conference ID1114.

[10] J. Barrios-Martí et al., for the KM3NeT Collaboration, proceedings of this conference ID1108.

[11] D. Stransky et al., for the KM3NeT Collaboration, proceedings of this conference ID1107.

[12] D. Gaggero et al., arXiv:1504.00227 (2015).

[13] M. Ahlers et al., arXiv:1505.03156 (2015).

[14] D. Gaggero et al., proceedings of this conference ID1126.

[15] F. Aharonian et al. (H.E.S.S. Collaboration), Astronomy \& Astrophysics 464 (2007) 235; corrigendum in Astronomy \& Astrophysics 531 (2011) C1.

[16] A. Abramowski et al. (H.E.S.S. Collaboration), Astronomy \& Astrophysics 548 (2012) A38.

[17] S.R. Kelner et al., Phys. Rev. D 74 (2006) 034018.

[18] F.L. Villante and F. Vissani, Phys. Rev. D 78 (2008) 103007; F. Vissani and F.L. Villante, Nucl. Instr. and Meth. A 588 (2008) 123; F. Vissani, Astropart. Phys. 26 (2006) 310.

[19] A. Trovato et al., for the KM3NeT Collaboration, proceedings of this conference ID1113.

[20] M. G. Aartsen et al. (IceCube Collaboration), Astroph. J. 796 (2014) 109.

[21] S. Adrián-Martínez et al. (ANTARES Collaboration), Astroph. J. Lett. L5 (2014) 786. 